\title{
Lobectomy by video-assisted thoracic surgery with mediastinal node sampling for lung cancer
}

This study was undertaken to determine whether a complete lung cancer operation can be done by video-assisted thoracic surgery and to evaluate the postoperative course. Forty-five patients (44 to 82 years of age) with clinical stage 1 lung cancer underwent video-assisted thoracic surgery for lobectomy or pneumonectomy with mediastinal lymph node sampling or dissection. There were no deaths or major complications. The median hospital length of stay was 4 days. Six patients stayed in the hospital for more than 7 days for pneumonia $(n=1)$, air leak $(n=3)$, or serous drainage $(n=2)$. Five of the seven patients who were 80 to 82 years of age were discharged by the fourth postoperative day. (J THORAC Cardiovasc Surg 1994;107:879-82)

Robert J. McKenna, Jr., MD, Los Angeles, Calif.

$\mathrm{V}$ ideo-assisted thoracic surgery (VATS) lobectomy potentially offers a significant decrease in morbidity compared with a thoracotomy for resection of lung cancer. A standard thoracotomy, or even a muscle-sparing thoracotomy, leads to considerable short- and long-term pain, as well as prolonged recovery time before return to work or full activity.

Lewis and associates ${ }^{1}$ reported the first thoracoscopic lobectomy with the use of standard instruments, a small incision, and thoracoscopy for visualization. Minimally invasive surgery has been shown to substantially decrease pain, morbidity, hospital length of stay, and return to work for a cholecystectomy. ${ }^{2}$ Perhaps video-assisted surgery can do the same for patients with lung cancer.

This report presents our initial experience with thoracoscopic lobectomy for lung cancer. The study was undertaken to evaluate the morbidity and mortality,

From the Department of Thoracic Surgery, University of Southern California School of Medicine, Los Angeles, Calif.

Read at the Nineteenth Annual Meeting of The Western Thoracic Surgical Association, Carlsbad, Calif., June 23-26, 1993.

Address for reprints: Robert J. McKenna, Jr., MD, 1245 Wilshire Blvd., Suite 606, Los Angeles, CA 90017-4807.

Copyright $\left.{ }^{(}\right) 1994$ by Mosby-Year Book, Inc.

$0022-5223 / 94 \$ 3.00+0 \quad \mathbf{1 2} / \mathbf{6} / \mathbf{5 2 2 2 6}$ length of stay, and the feasibility of performing a complete cancer operation with thoracoscopy.

\section{Patients and methods}

This study includes 45 consecutive patients ( $28 \mathrm{men}, 17$ women), 44 to 82 years of age (mean, 67 years) who underwent VATS lobectomy (Table I). Thirteen patients were over 70 years of age, and seven were over 80 years of age.

All patients had clinical stage 1 cancer without preoperative evidence of chest wall invasion, mediastinal adenopathy, or endobronchial tumor seen at bronchoscopy. Of the 45 tumors, 44 were less than $5 \mathrm{~cm}$ in size. One tumor was $6 \mathrm{~cm}$ in size.

The final pathologic staging is shown in Table II. During the operation, three tumors were found to be attached to the pericardum, and they were pathologically staged as T3. Two of these tumors were left upper lobe lesions that required a pericardial resection and an intrapericardial resection of the superior pulmonary vein. The other tumor was a left lower lobe lesion that required resection of the pericardium and phrenic nerve.

Preoperative computed tomographic scans showed normal mediastinal lymph nodes (less than $1 \mathrm{~cm}$ in size). All operations included mediastinal lymph node sampling or dissection. The final pathologic staging (Table I) showed two cases of microscopic N2 disease in lymph nodes that were grossly normal.

All procedures were performed while the patient was under general anesthesia with a double lumen tube and arterial line. With the patient in the lateral decubitus position, the rigid zero degree thoracoscope was passed through a $12 \mathrm{~mm}$ trocar in the seventh intercostal space in the anterior axillary line for an upper lobectomy or the midaxillary line for middle or lower lobecto- 
Table I. VATS resections

\begin{tabular}{lc}
\hline \multicolumn{1}{c}{ Resection } & No. of cases \\
\hline Right upper lobe & 10 \\
Right middle lobe & 6 \\
Right lower lobe & 8 \\
Right upper and middle lobes & 2 \\
Left upper lobe & 10 \\
Left lower lobe & 7 \\
Left pneumonectomy & 2 \\
\hline
\end{tabular}

my. The 30-degree lens was helpful for visualization of the superior aspect of the superior pulmonary vein and the pulmonary artery to the anterior segment of the upper lobe. A second camera in the second intercostal space midaxillary line was also used on two occasions, which was helpful for visualization of the superior aspect of hilar vessels during dissection.

Carbon dioxide inflation was used only to facilitate collapse of the lung and evacuation of smoke from electrocautery. The $12 \mathrm{~mm}$ trocar allowed maximal flow of carbon dioxide around the thoracoscope through the trocar.

No additional trocars were used. An access thoracotomy of 6 to $7 \mathrm{~cm}$ was made in various locations, but most commonly over the fourth interspace from the latissimus dorsi muscle to the pectoralis muscle. The ribs were not spread. A retractor was used if the chest wall was thick. Through this incision, standard pickups, scissors, and suction were used for dissection of the hilar vessels for an upper or middle lobectomy or in the fissure for a lower lobectomy. The dissection was easier for the surgeon standing anterior to the patient, rather than from the posterior of the patient, as for a standard posterolateral thoracotomy.

Additional $1 \mathrm{~cm}$ incisions were placed in varying locations for traction and countertraction. A 30-degree bend on the tip of the electrocautery facilitates visualization and dissection. A $1 \mathrm{~cm}$ incision in the fifth or sixth intercostal space in the midclavicular line provided the best angle for the vascular endo-GIA stapler (United States Surgical Corporation, Norwalk, Conn. [USSC]) to individually transect the appropriate branches of the pulmonary artery and vein. The knife blade was not removed. In some instances, traction on a tie around the artery or the use of a $5 \mathrm{~mm}$ mini-retractor (USSC) may facilitate placement of the endo-GIA stapler. The $3.5 \mathrm{~mm}$ endo-GIA stapler was used to transect the bronchus. The fissures were generally completed after the vessels and the bronchus were transected. The lung specimen was removed intact without morcellation. Mediastinal lymph nodes were routinely sampled (initially) or dissected (later, when more experience had been gained). In recent cases, at least 15 nodes (range 15 to 26 nodes) were examined. As the experience with VATS removal of mediastinal nodes progressed, complete dissection of nodes at all nodal stations was performed. The chest was drained with two straight $28 \mathrm{~F}$ chest tubes. The intercostal nerves were blocked with $0.5 \%$ bupivacaine hydrochloride (Marcaine) with epinephrine. Indomethacin (Indocin) suppositories were administered before the operation and continued after the operation for 48 hours.

\section{Results}

The operating time for the lobectomies averaged 125 minutes (range 45 to 240 minutes). During the time peri-
Table II. Final pathologic staging for the 45 cases of VATS lobectomy or pneumonectomy

\begin{tabular}{cc}
\hline $\begin{array}{c}\text { Stage of } \\
\text { resected tumors }\end{array}$ & $\begin{array}{c}\text { No. of } \\
\text { cases }\end{array}$ \\
\hline Stage I & \\
T1 N0 & 25 \\
T2 N0 & 12 \\
Stage II & \\
T1 N1 & 2 \\
T2 N1 & 1 \\
Stage III & \\
T3 N0 & 3 \\
T1 N2 & 1 \\
T2 N2 & 1 \\
\hline
\end{tabular}

od of this series, four additional procedures were begun with VATS and converted to a thoracotomy for safe dissection of granulomatous hilar nodes ( $n=1$ case), dissection of N1 disease $(n=1)$, to perform a sleeve resection after a VATS lobectomy because frozen section showed a positive bronchial margin $(n=1)$, and bleeding $(n=1)$. With further experience with the use of minimally invasive surgery, this bleeding could now be controlled with thoracoscopy without the need for thoracotomy.

More than 200 pulmonary arteries or veins were transected with the endo-GIA 30 vascular stapler (USSC). There was no significant problem with bleeding. In one case, a small amount of bleeding occurred from the end of a staple line. The stapler had been opened and closed multiple times on the artery before final positioning and firing. The site of the bleeding was oversewn with 5-0 Prolene sutures (Ethicon, Inc., Somerville, N.J.) without the need for a thoracotomy.

The largest tumor resected was a right upper lobe lesion measuring $6 \mathrm{~cm}$ in diameter. Removal was facilitated by resecting a $7 \mathrm{~cm}$ segment of rib at the incision through which the lobe was removed. This patient had no more pain than did the other patients in the series.

The median hospital length of stay was 4 days (range 2 to 16 days). Eleven patients were discharged on their second postoperative day, and 39 of $45(87 \%)$ were discharged by the fifth postoperative day.

There were no postoperative deaths. Complications included pneumonia $(n=1)$, prolonged serous drainage from the chest tube ( $n=2$, delaying discharge to day 13 and 15), and prolonged air leak ( $n=3$, delaying discharge to day 11,11 , and 16). The average blood loss was $440 \mathrm{ml}$, and two patients underwent transfusion.

Seven patients, 80 to 82 years of age, underwent VATS lobectomy $(n=6)$ or left pneumonectomy $(n=1)$. They 
were discharged on postoperative day 2, 3, 4, 4, 4, 11 (air leak), and 13 (prolonged serous drainage).

The patients in this series had considerably less pain after VATS lobectomy than did patients after our standard muscle-sparing thoracotomy incision. Indocin suppositories were used for 2 to 3 postoperative days. The patients had a moderate amount of pain on the day of the operation, but the pain decreased substantially on the first postoperative day. Only one patient had postthoracotomy syndrome, which resolved in 4 months. The most striking example of a benign course occurred in an 81-year-old woman who left the hospital on the second postoperative day without pain medication and promptly returned to full activity.

\section{Discussion}

This series represents a group of patients who underwent a VATS procedure to remove an intact lobe or lung and mediastinal lymph nodes. The pathology report showed that as many as 26 lymph nodes were removed (range 15 to 26 in recent cases). The goal to perform a standard lung cancer operation by VATS was accomplished.

The technique for the procedure evolved with more experience. Initially, the dissection was performed with laparoscopic instruments, and an incision to remove the lung specimen was made at the end of the procedure. Later, the access thoracotomy incision, proposed by Kirby and Rice ${ }^{3}$ was made as soon as the tumor was deemed resectable. This incision was used to accomodate standard thoracotomy instruments.

In contrast to the approach of Kirby and Rice, the access thoracotomy incision was made in the fourth intercostal space in the anterior axillary line, which placed the incision directly over the hilum. This technique allows easier dissection of the vessels and allows the procedure to be performed even if the fissures are poorly developed.

Lewis ${ }^{4}$ recently proposed the "cis-lobectomy," which really is a tourniquet lobectomy. The anterior location of our access thoracotomy makes individual ligation of the vessels and bronchi feasible.

Neither Kirby and Rice nor Lewis routinely resect mediastinal lymph nodes during a VATS lobectomy. Kirby and Rice routinely performed cervical mediastinoscopy to sample mediastinal nodes, but did not routinely remove mediastinal nodes with VATS. Lewis does not address mediastinal nodes in his paper. The current experience demonstrates that a complete cancer operation can be accomplished with VATS. The lung specimen is removed in a bag to minimize the risk of wound contamination. Longer follow-up is necessary to determine the 5-year survival of patients who undergo resection with VATS compared with those who undergo thoracotomy.

Alternative therapy for lung cancer in patients over 80 years of age has been used because of the risk of lobectomy or pneumonectomy in this age group. The seven patients over 80 in this study did very well. Perhaps VATS will allow conventional resections for elderly patients who are currently being denied the potential benefit of surgery.

Contraindications to VATS lobectomy include T2 tumors larger than $5 \mathrm{~cm}, \mathrm{~T} 3$ tumors invading the chest wall, centrally located tumors that can be seen in a lobar bronchus at bronchoscopy, sleeve resections, and prior neoadjuvant chemo-radiotherapy for stage IIIA nonsmall-cell lung cancer.

This study shows the feasibility of performing a complete cancer operation with VATS. Future refinement of techniques and instrumentation will simplify the procedure, and a larger series will be used to compare the morbidity, length of stay, and return to work after lobectomy by VATS with the results after thoracotomy.

\section{REFERENCES}

1. Lewis R, Caccavale RJ, Sisler GE, Mackenzie JW. One hundred consecutive patients undergoing video-assisted thoracic operations. Ann Thorac Surg 1992;54:421-6.

2. Stoker ME, Vose J, O'Mara P, Maini BS. Laparoscopic cholecystectomy: a clinical and financial analysis of 280 operations. Arch Surg 1992;127:589-96.

3. Kirby T, Rice T. Thoracoscopic lobectomy. Ann Thorac Surg 1993;56:784-6.

4. Lewis R. CIS-lobectomy. Presented at the Nineteenth Annual Meeting of The Western Thoracic Surgical Association; June 23-26, 1993; Carlsbad, Calif.

\section{Discussion}

Dr. John R. Benfield (Sacramento, Calif.). You have told us from your article, that thoracoscopic lobectomy potentially offers a significant decrease in morbidity compared with a thoracotomy. You have also told us that preoperative computed tomographic scans showed no suspicious lymph nodes, and that a 6 to $7 \mathrm{~mm}$ so-called access thoracotomy was made. This access incision was supplemented by port incisions resulting in approximately 9 to $10 \mathrm{~cm}$. The largest tumor that you resected required a $7 \mathrm{~cm}$ resection of a segment of rib. Your average hospital stay was 4.4 days, ranging from 2 to 15 , and 37 of 44 patients ( $89 \%$ ) were discharged by the fifth postoperative day. Were these really thoracoscopic lobectomies or was this video-assisted thoracic surgery?

My experience with thoracoscopy is more than 30 years, but I have used modern video-assisted thoracic surgery in only 57 selected cases ( $27 \%$ ) of potentially eligible patients. In about 21 of my patients (37\%), I proceeded either by plan or, because of the findings, to a standard rib-spreading thoracotomy. Videoassisted exploration was helpful in some way in practically all of these 57 cases.

I would have preferred to see you entitle this important pre- 
sentation, "Lobectomy by Video-Assisted Thoracic Surgery in Selected Lung Cancer Patients."

My first question concerns the denominator for your videoassisted lobectomy experience. In other words, during the period about which you are reporting, how often did you proceed to traditional rib-spreading thoracotomy after preliminary videoassisted exploration? And how many lobectomies for cancer were done by traditional means during the period of your reporting?

Dr. McKenna: You mentioned taking out the seventh rib. I wanted to clarify that point. There was a series from Germany in which a rib was removed for larger tumors; I thought I would try that in one case, and it was a larger tumor. In general, I made the $6 \mathrm{~cm}$ incision. No rib spreader was used, and the ribs were not spread apart. This is really a VATS case, and I agree that I probably should call it a VATS lobectomy rather than a thoracoscopic lobectomy.

With regard to the selection at this point in time, I attempt to take out all stage I lung tumors with thoracoscopy. During this 45 case series, there were four cases that I converted to thoracotomy, but all the rest were done with thoracoscopy. It certainly is not the appropriate procedure for every case of lung cancer. I would not use this procedure for Pancoast's tumors or a tumor that required a sleeve resection, in particular, because of the dissection around the blood vessels and the difficulty of deciding between a pneumonectomy or a sleeve resection. I would not use this procedure for patients with mediastinoscopy proven N2 disease who, before the operation, had received chemotherapy and radiation. I think I need to do a thoracotomy to safely do that operation and safely do the dissection. But, for the routine stage I lung cancer, this is my operation of choice at this point, and I am able to complete $90 \%$ of them -45 with this method and four I converted.

Dr. Benfield. When I read your manuscript I reviewed the decision tree that I follow during and around operations to treat lung cancer. The importance of palpation of the lung and the mediastinum came immediately to mind with a view to searching for previously unsuspected lesions, and for the purpose of assessing the advisability of segmental resections or segmentectomy. I then reviewed the anatomic issues that are peculiar and potentially hazardous pertaining to each of the five lobectomy procedures. Recurrent themes were proximal vascular control and small but short and potentially hazardous vessels such as the posterior ascending vessel of the right upper lobe. Another issue is the B6 or superior segment artery and bronchus and how these structures relate to optimum dissection and transection of the lower lobe, middle lobe, and lingula bronchi. And finally, I recalled a few recent cases that required suture methods in preference to the use of stapling machines. This brings me to my next question. Which of the lobectomies, if any, has been easiest, and which has been most difficult with the use of exclusively video-assisted means?

Dr. McKenna. You bring up a couple of good points about other cases that are not appropriate for a VATS lobectomy: a centrally located tumor with a proximity to the blood vessels and tumors that you can see with the bronchoscope within the mainstem or lobar bronchus certainly are not appropriate, so I agree with what you are saying about patient selection for this type of procedure.
I have not found one lobe to be easier or more difficult. The whole experience is evolving, and it is a matter of figuring out the proper angles. I think a big difference with the way I have done these procedures, compared with the way Dr. Kirby has done them, is making that incision farther anteriorly and superiorly. His incision is lower so you are still working up and around, and I think it is more difficult to do it that way. He also proceeds posteriorly for a right upper lobe to first transect the bronchus and then the blood vessels. He has trouble with patients with complete fissures. I am certainly not saying that incomplete fissures are necessarily easy, but when you operate from the front, when you get the blood vessels first and the last maneuver is the fissure, then I think it is easier.

Dr. Benfield. How about segmentectomies?

Dr. McKenna. I have not done any segmentectomies, and I think the word is still out on whether a wedge or a segment is as good as a lobectomy. My bias is that a lobectomy is the standard treatment for lung cancer: that is what should be done, and that is what I do. I cannot feel the mediastinum for enlarged nodes. I certainly inspect the whole chest first, and I remove those lymph nodes; thus, I think the patients who benefit from operation for N2 disease are the ones with no enlarged lymph nodes. You have done the node dissection, and you are surprised when the pathology report shows $\mathrm{N} 2$ disease; those are the patients with stage III disease for whom I use this type of resection.

Dr. John E Connolly. (Irvine, Calif.). I commend you for the leadership you have shown.

Dr. Walter Cannon (Palo Alto, Calif.). I would like to ask Dr. McKenna a couple of questions. One is whether he is getting any response from the instrument and stapling manufacturers on getting the proper angle for dividing these vessels. One of the obvious problems that he has had in doing this procedure is that he is never quite at the right angle to put the stapling device on the vessel.

Dr. McKenna. I think part of that is experience doing the procedure and trying to figure out the angles. For most of the vessels, the best angle is to go in the fifth or sixth intercostal space in the midclavicular line, and you can usually manipulate the staple from that angle to be perpendicular to most of the vessels. Certainly if an instrument reticulated up and down it would make it a lot easier for us.

Dr. Cannon. The second question I have is, those of us who are in teaching institutions working with residents feel somewhat uncomfortable doing this procedure with a resident who may or may not be experienced in the technique. What is your opinion about teaching residents to do this procedure, particularly for those of us who have not done a lot of them?

Dr. McKenna. I certainly feel uncomfortable about having a resident do one of these cases himself or herself at this point. They assist me in the cases, and the video skills of surgeons and residents vary greatly: some have no experience at all, and others have pretty good video skills. As my experience grows and I develop and improve the techniques for the different lobes, I think I will be able to get to the point where I can prepare everything so that it could be done by a resident. I think an important aspect is making the incision anteriorly. I stand in front of the patient just, as when I use a standard muscle-sparing thoracotomy. 